\title{
Weak association between subjective symptoms of and objective testing for dry eyes and dry mouth: results from a population based study
}

\author{
Elaine M Hay, E Thomas, B Pal, Ali Hajeer, H Chambers, A J Silman
}

\begin{abstract}
Objectives-To determine associations between symptoms of dry eyes and dry mouth and objective evidence of lacrimal and salivary gland dysfunction in a population based sample. To determine associations between these elements and the presence of autoantibodies.

Methods-A cross sectional population based survey. Subjects were interviewed and examined (Schirmer-1 test and unstimulated salivary flow) for the presence of dry eyes and mouth. Antibodies (anti-Ro [SS-A], anti-La [SS-B], rheumatoid factor, antinuclear antibody) were measured.

Results-341 subjects were examined. Twenty four per cent had dry eye symptoms, $29 \%$ dry mouth symptoms, and $14 \%$ both. There was only a weak association between the presence of oral or ocular symptoms and their respective test results. Associations were strongest between dry mouth symptoms and positive test results, and in subjects under 55 years of age. There was no association between the presence of autoantibodies and either symptoms or signs of dry eyes or dry mouth.

Conclusion-Only weak associations were found between self reported symptoms of dry eyes and dry mouth and objective measures said to define Sjögrens syndrome in the general population. The clinical significance of these symptoms in the community needs reappraisal. (Ann Rheum Dis 1998;57:20-24)
\end{abstract}

Staffordshire

Rheumatology Centre, Stoke on Trent

E M Hay

ARC Epidemiology

Research Unit,

Manchester

E Thomas

A Hajeer

H Chambers

A J Silman

Withington Hospital, Manchester

B Pal

Correspondence to:

Dr E M Hay, Staffordshire

Rheumatology Centre, High

Lane, Burslem, Stoke on

Trent, ST6 7AG.

Accepted for publication

13 November 1997 commonly used in clinical practice, is Schirmer's test, which measures the volume of tears produced in five minutes under basal condi-
Most of the commonly used tests of glandular function measure either quantity or quality of tears or saliva produced. The simplest test for aqueous deficient dry eye, and the one most
The term Sjögren's syndrome describes a hetcrogeneous group of autoimmune disorders
characterised by inflammation affecting predominantly the exocrine glands, resulting in mouth. Classification of Sjögren's syndrome criteria sets, using differing combinations of oral and ocular symptoms and tests for dry eyes and mouth, have been proposed. criteria require the presence of ocular and oral symptoms in addition to objective evidence of tions using a standard strip of paper placed under the subject's lower eye lid. ${ }^{2}$ Schirmer's test has been shown to perform well in the hospital environment, ${ }^{3}$ with sensitivity of 0.77 and specificity of 0.72 against the physicians' "gold standard" diagnosis of Sjögren's syndrome. However, one small community study reported poor reproducibility of Schirmer's test when used to screen normal subjects, ${ }^{4}$ leading the authors to conclude that the test was of little clinical value in screening for the presence of dry eyes. The only two alternative tests that may be used for assessing lacrimal function are the van Bijsterveld score and the tear break up time. The van Bijsterveld score measures the number of dead cells on the corneal and conjunctival surfaces after instillation of rose bengal staining. Although it has been shown to be a good test for evaluating dry eyes, ${ }^{5}$ it is very uncomfortable for the subject and hence only appropriate for use in hospital settings. Break up time measures the stability of the tear film. ${ }^{2}$ It is easy to perform but requires the use of a slit lamp and is therefore limited to ophthalmological practice.

With regard to assessment of dry mouth, the test thought to be most representative for measuring under normal basal conditions is the unstimulated whole salivary flow. ${ }^{1}$ Ideally it should be measured over at least 15 minutes, between 9 and 11 am and with no smoking, food or drink for at least one hour before the test. ${ }^{2}{ }^{6}$ However, in both clinical practice and the research setting, such precision is difficult to achieve. Of the alternative tests of oral function, the whole stimulated salivary flow, measured over five minutes has produced inconsistent results because of a lack of agreement about how the test should be performed. ${ }^{1}$ Other tests for measuring salivary gland function are either invasive (minor salivary gland biopsy and sialography) or require special equipment (scintigraphy or magnetic resonance scanning) making them unsuitable for use in population based studies.

In conclusion, although no single test of ocular or salivary gland function is sufficiently precise to diagnose Sjögrens syndrome, in hospital settings Schirmer-1 test and unstimulated salivary flow test seem to perform well. ${ }^{378}$ Furthermore, these are the only two tests feasible to use in large scale epidemiological studies.

We therefore examined associations between symptoms of dry eyes and dry mouth and abnormalities of Schirmer-1 test and unstimulated salivary flow test in a general population 
Table 1 Symptom frequency, sex, and age difference between study participants and non-participants

\begin{tabular}{|c|c|c|c|}
\hline & Participants $(n=341)$ & Non-participants $(n=275)$ & p Value \\
\hline \multicolumn{4}{|c|}{ Sex (frequency (\%)) } \\
\hline Male & $152(44.6)$ & $140(50.9)$ & $\mathrm{p}=0.118^{\star}$ \\
\hline Female & $189(55.4)$ & $135(49.1)$ & \\
\hline \multicolumn{4}{|c|}{ Age (median (IQR)) } \\
\hline Male & $46(35,57)$ & $40(29,55)$ & $\mathrm{p}=0.044 \dagger$ \\
\hline Female & $49(35,59)$ & $38(28,60)$ & $\mathrm{p}=0.005 \dagger$ \\
\hline \multicolumn{4}{|c|}{$\begin{array}{l}\text { Ocular symptoms (frequency }(\%)) \\
\quad \text { (from general health survey } \ddagger)\end{array}$} \\
\hline Yes & $96(30.2)$ & $45(16.6)$ & $\mathrm{p}<0.0001^{\star}$ \\
\hline No & $222(69.8)$ & $226(83.4)$ & \\
\hline \multicolumn{4}{|c|}{$\begin{array}{l}\text { Oral symptoms (frequency (\%)) } \\
\text { (from general health survey } \ddagger)\end{array}$} \\
\hline Yes & $85(26.7)$ & $52(19.2)$ & $\mathrm{p}=0.033^{\star}$ \\
\hline No & $234(73.3)$ & $219(80.8)$ & \\
\hline
\end{tabular}

${ }^{\star} \mathrm{p}$ Value, $\chi^{2}$ test. $\nmid \mathrm{p}$ Value, Mann-Whitney test. $\ddagger$ Frequencies do not add to totals because of missing data.

sample to discover if their co-occurrence in hospital patients with Sjögrens syndrome is reflected in the community. In addition we investigated the associations between these elements and the presence of autoantibodies (rheumatoid factor (RF), antinuclear antibody (ANA), antibodies to Ro or La).

\section{Methods}

Subjects recruited to the study were respondents to a cross sectional general health survey, which was performed on 1000 adults aged 18 to 75 years randomly selected from a population register of people from a local general practice. This survey was part of a large population based study mainly focused on musculoskeletal disorders. Respondents to the first wave (610 subjects) were asked whether they would consent to a home visit by a research nurse as part of a study into dry eyes and dry mouth. The general health survey included some broad general questions on dry eyes and dry mouth. Thus it was possible to use these data to compare those agreeing to with those declining a home visit, to assess the extent of any non-participation bias.

At the home visit a detailed questionnaire covering symptoms of dry eyes and dry mouth, plus questions about demographic details and medical history was completed. The questionnaire included the following questions, relating to the previous three months, which were used to classify subjects as having dry eyes or dry mouth $^{7}$ (see table 2):
1 (a) Do you suffer from troublesome dry eyes every day?

(b) Do you have a recurrent sensation of sand or gravel in your eyes?

(c) Do you use artificial tears more than three times a day?

2 (a) Does your mouth feel dry every day?

(b) Have you had recurrent or persistent swelling of your salivary glands as an adult?

(c) Do you frequently have to take a drink in order to swallow?

A single positive statement in each category classified a case.

The nurse performed a Schirmer-1 test and measured unstimulated whole salivary flow (USF). For Schirmer's test, wetting of $5 \mathrm{~mm}$ or less of the standard Sno-strip paper (Smith and Nephew) measured over five minutes under basal conditions with the eyes lightly closed was considered abnormal. ${ }^{2}$ For USF, a shortened version of a previously described technique, ${ }^{2}$ more suitable for use in community surveys was used, in which a saliva volume of $0.5 \mathrm{ml}$ or less, collected into a universal container for five minutes under basal conditions and measured using a $2 \mathrm{ml}$ insulin syringe, was considered abnormal. Subjects were asked to refrain from eating, drinking or smoking for at least one hour before the test.

A blood sample was obtained for measurement of antibodies to Ro and La by ELISA (Shield Diagnostics), ANA by indirect immunofluorescence, and RF by Latex test. The

Table 2 Frequencies of ocular and oral symptoms

\begin{tabular}{|c|c|c|c|}
\hline & $\begin{array}{l}\text { Men }(n=152) \dagger \\
\text { Frequency (\%) with } \\
\text { symptom }\end{array}$ & $\begin{array}{l}\text { Women }(n=189) t \\
\text { Frequency }(\%) \text { with } \\
\text { symptom }\end{array}$ & $\begin{array}{l}\text { Overall }(n=341) t \\
\text { Frequency }(\%) \text { with } \\
\text { symptom }\end{array}$ \\
\hline Dry eyes $\star$ & $9(5.9)$ & $26(13.8)$ & $35(10.3)$ \\
\hline Sand or gravel in eyes* & $23(15.1)$ & $52(27.5)$ & $75(22.0)$ \\
\hline Use artificial tears $>3$ times daily ${ }^{\star}$ & $1(0.7)$ & $5(2.7)$ & $6(1.8)$ \\
\hline Itching of eyes & $47(30.9)$ & $72(38.1)$ & $119(34.9)$ \\
\hline Redness or inflammation of eyes & $24(15.8)$ & $29(15.5)$ & $53(15.6)$ \\
\hline Eye lids stuck together in morning & $9(5.9)$ & $19(10.1)$ & $28(8.2)$ \\
\hline Smoke irritates eyes & $80(52.6)$ & $117(62.6)$ & $197(58.3)$ \\
\hline Burning sensation in eyes & $11(7.2)$ & $21(11.2)$ & $32(9.5)$ \\
\hline Feels as if "something in eyes" & $34(22.4)$ & $68(36.8)$ & $102(30.3)$ \\
\hline Light hurts eyes & $40(26.3)$ & $60(35.7)$ & $100(29.6)$ \\
\hline Positive ocular symptoms ${ }^{\star}$ & $25(16.5)$ & $56(29.8)$ & $81(23.8)$ \\
\hline Dry mouth^ & $22(14.5)$ & $42(22.3)$ & $64(18.8)$ \\
\hline Persistent swelling of glands ${ }^{\star}$ & $4(2.7)$ & $4(2.1)$ & $8(2.4)$ \\
\hline Drink to swallow food ${ }^{\star}$ & $19(12.5)$ & $33(17.5)$ & $52(15.3)$ \\
\hline Use saliva substitutes $>3$ times daily & $1(0.7)$ & $2(1.1)$ & $3(0.9)$ \\
\hline Take drink to bed & $25(16.5)$ & $39(20.6)$ & $64(18.8)$ \\
\hline Positive ocular symptoms ${ }^{\star}$ & $40(26.5)$ & $57(30.3)$ & $97(28.6)$ \\
\hline Positive ocular and oral symptoms ${ }^{\star}$ & $13(8.6)$ & $35(18.7)$ & $48(14.2)$ \\
\hline
\end{tabular}

${ }^{\star}$ Questions used for classification of subjects (according to Vitali et al Arthritis Rheum 1993;36:340-7). †Frequencies do not add to totals because of missing data. 


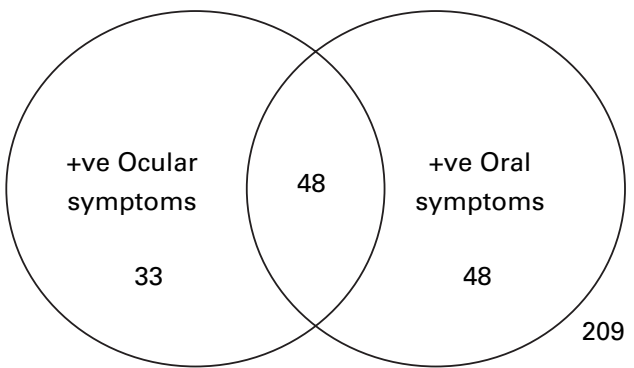

Figure 1 Combinations of ocular and oral symptoms. Numbers represent subjects classified as having symptoms of dry eyes (ocular) and dry mouth (oral).

following values were defined as abnormal: anti-Ro and anti-La $>2 \mathrm{U} / \mathrm{ml}, \mathrm{RF}>1: 40$, ANA $>1: 120$.

ANALYSIS

The relation between the subjective symptoms and the objective tests was analysed by calculating (a) positive predictive value (PPV) defined as the proportion of subjects with symptoms who had a positive objective test, (b) negative predictive value (NPV) defined as the proportion of subjects without symptoms who had a negative objective test. The likelihood ratio (LR) and the 95\% confidence intervals were then calculated for each association and can be interpreted as the increased likelihood of being positive on an objective test, given the presence of symptoms. A value less than unity indicates symptoms being less predictive than random of a positive test result. Analyses were conducted separately for Schirmer-1 test, USF, and the presence of autoantibodies. As there was no difference in the results of associations between men and women, data from both sexes were pooled for analysis. By contrast, age was an important confounder and thus data were also analysed stratifying the population into those aged above and below 55 years.

\section{Results}

In all, 341 (55\%) of the 610 subjects approached agreed to be interviewed. Participants were more likely to be women and older than non-participants and were more likely to have answered positively to the general health survey questions about dry eyes and dry mouth (table 1).

Table 3 Associations between ocular and oral symptoms and the respective objective test results

\begin{tabular}{|c|c|c|c|c|c|}
\hline & \multicolumn{5}{|c|}{ Abnormal Schirmer-1 test? } \\
\hline & Yes & No & $P P V^{\star}$ & $N P V^{\star}$ & $L R(95 \% C I) \dagger$ \\
\hline \multicolumn{6}{|c|}{ +ve Ocular symptoms? } \\
\hline Yes & 19 & 57 & \multirow[t]{2}{*}{0.25} & \multirow[t]{2}{*}{0.77} & \multirow{2}{*}{$\begin{array}{l}1.10 \\
(0.7,1.7)\end{array}$} \\
\hline No & 51 & 175 & & & \\
\hline \multicolumn{6}{|c|}{ +ve Ocular and oral symptoms? } \\
\hline Yes & 13 & 33 & \multirow[t]{2}{*}{0.28} & \multirow[t]{2}{*}{0.78} & \multirow{3}{*}{$\begin{array}{l}1.29 \\
(0.7,2.3)\end{array}$} \\
\hline \multirow[t]{2}{*}{ No } & 57 & 197 & & & \\
\hline & \multicolumn{4}{|c|}{ Abnormal unstimulated saliva flow rate? } & \\
\hline \multicolumn{6}{|c|}{ +ve Oral symptoms? } \\
\hline Yes & 38 & 53 & \multirow[t]{2}{*}{0.42} & \multirow[t]{2}{*}{0.76} & \multirow{2}{*}{$\begin{array}{l}1.77 \\
(1.3,2.5)\end{array}$} \\
\hline No & 55 & 177 & & & \\
\hline \multicolumn{6}{|c|}{ +ve Ocular and oral symptoms? } \\
\hline Yes & 25 & 22 & \multirow[t]{2}{*}{0.53} & \multirow[t]{2}{*}{0.75} & \multirow{2}{*}{$\begin{array}{l}2.80 \\
(1.7,4.6)\end{array}$} \\
\hline No & 68 & 207 & & & \\
\hline
\end{tabular}

$\star \mathrm{PPV}$, positive predictive value, $\mathrm{NPV}$, negative predictive value

†, LR (95\% CI)=likelihood ratio (95\% confidence intervals).

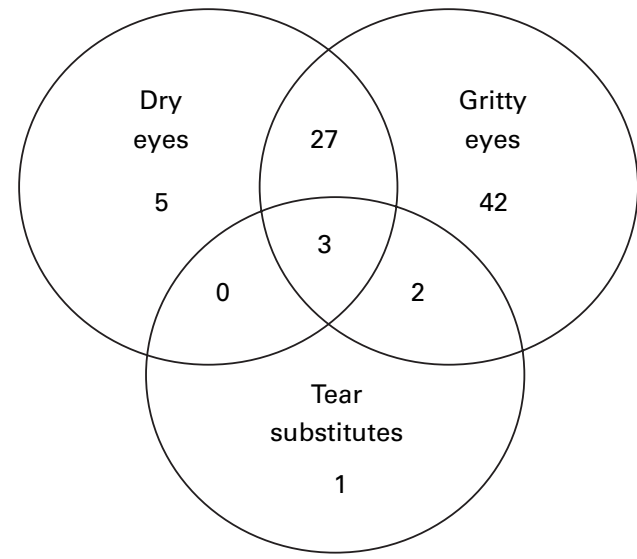

Figure 2 Combination of ocular symptoms. Numbers represent subjects answering positively to each of the questions about dry eyes and dry mouth listed in table 1. "Dry eyes"= question 1a; "gritty eyes"= question $1 b$; "tear substitutes" = question 1 c.

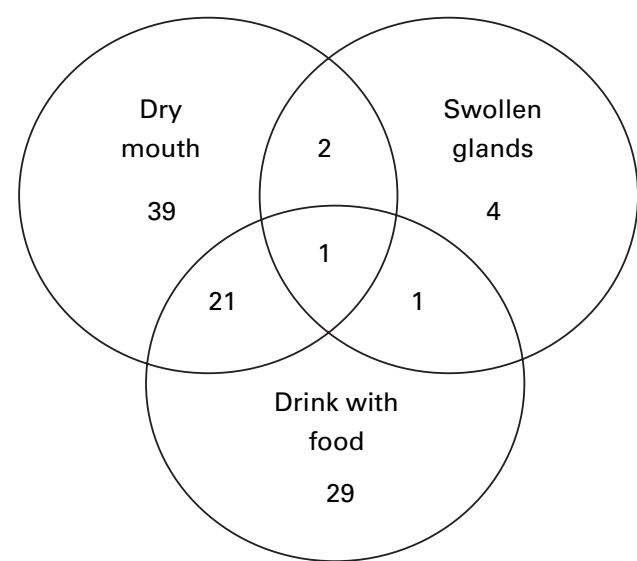

Figure 3 Combination of oral symptoms. "Dry mouth"= question 2a; "swollen glands"= question $2 b$; "drink with food"= question $2 c$.

OCULAR AND ORAL SYMPTOMS

Table 2 shows the percentage of subjects reporting various oral and ocular symptoms at interview. Using the questions proposed by Vitali et al, ${ }^{7} 81$ (24\%) subjects had one or more dry eye symptoms (25 (17\%) men, 56 (30\%) women) and 97 (29\%) one or more dry mouth symptoms (40 (27\%) men, 57 (30\%) women). Forty eight (14\%) of these reported both dry eyes and dry mouth symptoms (13 (9\%) men, 35 (19\%) women) (fig 1).

Overall the most common ocular symptom was "smoke irritates the eyes", which was reported by $58 \%$. Considering the three ocular questions used for classification purposes, "sand or gravel sensation in the eyes" was the most commonly reported (22\%). Furthermore, $93 \%$ of those subsequently classified as having dry eyes reported that symptom. Only $4 \%$ of subjects answered positively to all three dry eye questions (fig 2).

The most commonly reported oral symptom was "dry mouth every day", present in $18 \%$ of subjects overall and $66 \%$ of the subgroup classified as having "dry mouth" symptoms. Although some subjects answered positively to more than one dry mouth question, only $1 \%$ reported all three (fig 3). 
Table 4 Associations between ocular symptoms and Schirmer-1 test: subjects aged under and over 55 years

\begin{tabular}{|c|c|c|c|c|c|c|c|c|c|c|}
\hline & \multicolumn{10}{|c|}{ Abnormal Schirmer-1 test? } \\
\hline & \multicolumn{5}{|c|}{ Subjects aged under 55 years } & \multicolumn{5}{|c|}{ Subjects aged over 55 years } \\
\hline & Yes & No & $P P V$ & $N P V$ & $\begin{array}{l}L R \\
(95 \% C I)^{*}\end{array}$ & Yes & No & $P P V$ & $N P V$ & $\begin{array}{l}L R \\
(95 \% C I)^{*}\end{array}$ \\
\hline \multicolumn{11}{|l|}{ +ve Ocular symptoms? } \\
\hline Yes & 13 & 32 & 0.29 & 0.84 & 1.76 & 6 & 25 & 0.19 & 0.62 & 0.50 \\
\hline $\begin{array}{l}\text { No } \\
\text { +ve Ocular and oral symptoms? }\end{array}$ & 25 & 133 & & & $(1.3,2.3)$ & 26 & 42 & & & $(0.3,0.7)$ \\
\hline Yes & 8 & 19 & 0.30 & 0.83 & 1.81 & 5 & 14 & 0.26 & 0.66 & 0.75 \\
\hline No & 30 & 144 & & & $(1.1,3.0)$ & 27 & 53 & & & $(0.2,3.2)$ \\
\hline
\end{tabular}

${ }^{\star} \mathrm{LR}(95 \% \mathrm{CI})=$ likelihood ratio $(95 \%$ confidence intervals $)$. Abbreviations as in Table 3.

Table 5 Associations between oral symptoms and unstimulated saliva flow test: subjects aged under and over 55 years

\begin{tabular}{|c|c|c|c|c|c|c|c|c|c|c|}
\hline & \multicolumn{10}{|c|}{ Abnormal unstimulated saliva flow rate? } \\
\hline & \multicolumn{5}{|c|}{ Subjects aged under 55 years } & \multicolumn{5}{|c|}{ Subjects aged over 55 years } \\
\hline & Yes & No & $P P V$ & $N P V$ & $\begin{array}{l}L R \\
(95 \% C I)^{*}\end{array}$ & Yes & No & $P P V$ & $N P V$ & $\begin{array}{l}L R \\
(95 \% C I)^{*}\end{array}$ \\
\hline \multicolumn{11}{|l|}{ +ve Oral symptoms? } \\
\hline Yes & 19 & 33 & 0.37 & 0.84 & 2.20 & 19 & 20 & 0.49 & 0.58 & 1.20 \\
\hline No & 25 & 135 & & & $(1.9,2.5)$ & 30 & 42 & & & $(0.6,2.4)$ \\
\hline +ve Ocular and oral symptoms? & & & & & & & & & & \\
\hline Yes & 13 & 13 & 0.50 & 0.83 & 3.82 & 12 & 9 & 0.57 & 0.58 & 1.66 \\
\hline No & 31 & 155 & & & $(3.2,4.5)$ & 37 & 52 & & & $(0.9,3.0)$ \\
\hline
\end{tabular}

${ }^{\star} \mathrm{LR}(95 \% \mathrm{CI})=$ likelihood ratio (95\% confidence intervals). Abbreviations as in Table 3.

OBJECTIVE ORAL AND OCULAR TESTS

USF was reduced, indicating abnormal dryness of the mouth, in $29 \%$ of subjects tested. Schirmer-1 test demonstrated reduced tear production in $23 \%$ of subjects tested. Thirty five of 297 subjects tested (12\%) were abnormal for both tests.

There was a only a weak association between the presence of oral or ocular symptoms and their respective test results (table 3 ). The association between oral symptoms and USF was stronger than that between ocular symptoms and Schirmer-1 test. Subjects with both ocular and oral symptoms showed the strongest associations with the objective test results.

Associations were examined separately for subjects under and over 55 years of age in view of higher frequencies of abnormal findings reported in the elderly. ${ }^{9}$ Both ocular and oral symptoms were more likely to be associated with abnormal test results in younger than older subjects (tables 4 and 5).

\section{PRESENCE OF AUTOANTIBODIES}

Table 6 shows the frequencies of autoantibodies detected in 271 subjects who consented to a blood test, which were similar in men and women. There was, however, no evidence of any important associations between the presence of autoantibodies and either symptoms or objective

Table 6 Presence of autoantibodies

\begin{tabular}{llll}
\hline Presence of autoantibodies & $\begin{array}{l}\text { Men }(n=122)^{*} \\
\text { Positive (\%) }\end{array}$ & $\begin{array}{l}\text { Women }(n=149) * \\
\text { Positive (\%) }\end{array}$ & $\begin{array}{l}\text { All }(n=271)^{*} \\
\text { Positive (\%) }\end{array}$ \\
\hline +ve anti-Ro & $15(12.3)$ & $14(9.4)$ & $29(10.7)$ \\
+ve anti-La & $4(3.3)$ & $6(4.0)$ & $10(3.7)$ \\
+ve anti-Ro or anti-La & $16(13.1)$ & $16(10.7)$ & $32(11.8)$ \\
+ve ANA & $6(5.2)$ & $17(11.6)$ & $23(8.8)$ \\
+ve Rheumatoid Factor & $4(3.5)$ & $7(4.8)$ & $11(4.2)$ \\
Any +ve Autoantibodies & $23(18.9)$ & $33(22.2)$ & $56(20.7)$ \\
\hline
\end{tabular}

${ }^{\star}$ Frequencies do not add to 341 because of incomplete collection and analysis of some blood samples.

+ve anti-Ro $=\geqslant 2$ international units $/ \mathrm{ml}$. +ve anti- $\mathrm{La}=\geqslant 2$ international units $/ \mathrm{ml}$. +ve ANA= $>1: 120$. +ve $\mathrm{RF}=>1: 40$. signs of dry eyes or dry mouth (table 7 ). This was true both for individual autoantibodies as well as for the presence of any autoantibody across all age groups.

\section{Discussion}

In this population based study both symptoms and signs suggestive of dry eyes and dry mouth were common. Surprisingly, however, subjects with such symptoms were only slightly more likely than those without to have an abnormal Schirmer-1 or USF test result. USF seemed to perform better than Schirmer-1 test in this respect; the strongest association was between oral symptoms and abnormal USF in younger subjects. In addition, the presence of antibodies to Ro or La, commonly associated with Sjögren's syndrome in hospital patients ${ }^{10-12}$ was neither associated with symptoms nor abnormal test results in this community sample.

The findings from this population based study suggest that the ocular and oral questions we examined did not identify a subgroup of subjects more likely to have abnormalities on objective testing of lacrimal and salivary flow. There are several possible explanations for our findings. Firstly, the low prevalence of affected subjects in our sample (compared with hospital samples) will lead to a high number of false positive test results, thus leading to a low predictive value. Secondly, the milder spectrum of disease seen in the community and the fact that standardisation of the tests is more difficult when they are not performed under hospital conditions may reduce the sensitivity and specificity of the objective tests. Thirdly, the precision of measurements obtained using Schirmer's test and USF test are likely to be lower when they are used in a community setting compared with a hospital clinic, thereby increasing measurement error. For example, one of the limitations of our study was that we found it impossible to standardise 
Table 7 Associations between the presence of autoantibodies and symptoms and objective tests

\begin{tabular}{|c|c|c|c|c|c|}
\hline & \multicolumn{2}{|c|}{ Autoantibodies present? } & \multirow[b]{2}{*}{$P P V$} & \multirow[b]{2}{*}{$N P V$} & \multirow[b]{2}{*}{$L R(95 \% C I) t$} \\
\hline & Yes $(n=56)^{\star}$ & No $(n=215)^{\star}$ & & & \\
\hline \multicolumn{6}{|c|}{ +ve Ocular symptoms? } \\
\hline Yes & 14 & 48 & \multirow[t]{2}{*}{0.23} & \multirow{2}{*}{0.80} & \multirow[t]{2}{*}{$1.11(0.7,1.9)$} \\
\hline No & 42 & 166 & & & \\
\hline \multicolumn{6}{|c|}{ +ve Oral symptoms? } \\
\hline Yes & 17 & 65 & \multirow[t]{2}{*}{0.21} & \multirow[t]{2}{*}{0.79} & \multirow[t]{2}{*}{$0.99(0.6,1.6)$} \\
\hline No & 39 & 148 & & & \\
\hline \multicolumn{6}{|c|}{ +ve Ocular and oral symptoms? } \\
\hline Yes & 9 & 29 & \multirow[t]{2}{*}{0.24} & \multirow[t]{2}{*}{0.80} & \multirow[t]{2}{*}{$1.17(0.6,2.3)$} \\
\hline No & 47 & 183 & & & \\
\hline \multicolumn{6}{|c|}{ +ve Schirmer-1 test? } \\
\hline Yes & 12 & 49 & \multirow[t]{2}{*}{0.19} & \multirow[t]{2}{*}{0.78} & \multirow[t]{2}{*}{$0.91(0.5,1.6)$} \\
\hline No & 41 & 147 & & & \\
\hline \multicolumn{6}{|c|}{ +ve USF test? } \\
\hline Yes & 15 & 62 & \multirow[t]{2}{*}{0.19} & \multirow[t]{2}{*}{0.78} & \multirow[t]{2}{*}{$0.91(0.6,1.5)$} \\
\hline No & 41 & 148 & & & \\
\hline
\end{tabular}

${ }^{\star}$ Frequencies do not add to totals because of missing data.

†LR $(95 \% \mathrm{CI})=$ likelihood ratio $(95 \%$ confidence intervals). Abbreviations as in Table 3.

the time of day the USF test was performed, and although patients were requested not to eat or drink for one hour before the test this was also sometimes difficult to avoid. Similarly, a 15 minute collection time for the saliva proved too lengthy for inclusion in a large scale population study and was not acceptable to the study subjects. The effect of performing the USF test immediately after an interview lasting approximately 15 minutes (the blood test was performed last of all) is also not known. These methodological and practical problems will all reduce the level of associations between questions about dry eyes and dry mouth and the respective test results in our study.

We confirmed the previously reported higher frequency of ocular and oral symptoms and positive test results on older subjects. ${ }^{9}$ This is thought to be caused by age related changes in exocrine glands leading to lacrimal and salivary hypofunction. It is interesting to note that the association between the symptoms of dry eyes and abnormal Schirmer test is even weaker in this group than in younger subjects: older subjects with ocular symptoms were less likely than their younger counterparts to have an abnormal Schirmer test. It is probable that ocular symptoms result from causes other than dryness, such as anterior or posterior blepharitis, in the elderly.

One previous study has compared various measures of exocrine gland function with levels of antibodies to Ro and $\mathrm{La}$ in a population sample. ${ }^{13}$ In contrast with our findings, subjects with symptoms of dry eyes or dry mouth were found to have relatively impaired exocrine gland function on objective testing, and also had higher levels of antibodies to Ro and La. Reported differences were small, however, and may not be of clinical significance (for example, sum of right and left eye-Schirmer test $27.6 \mathrm{~mm}$ in symptomatic subjects compared with $26.4 \mathrm{~mm}$ in controls).

Only $55 \%$ of subjects approached agreed to participate in our study. We considered the possible effect of selection bias on our findings. Although those participating had a higher frequency of symptoms of dry eyes and dry mouth, it is unlikely that the associations between symptoms and objective test results would differ significantly in those who participated compared with those who did not. Intuitively it might be expected that associations would be, if anything, stronger in the participating than the non-participating group. We, therefore, feel that it is unlikely that our study will have underestimated the level of associations.

In summary, we found only weak associations between self reported symptoms of dry eyes and dry mouth with objective measures of lacrimal and salivary gland functions, in the general population. The clinical significance of dry eyes and dry mouth in the community needs further appraisal.

We would like to thank the general practitioners and their patients for participating in this study, and the Arthritis and patients for participating in this study, and the Arthri
Rheumatism Council (UK) for its financial support.

1 Manthorpe R, Jacobsson LTH. Classification and assessment of rheumatic diseases: Part II: Sjögrens syndrome. Ballieres Clin Rheumatol 1995;9:483-96.

2 Workshop participants. Manual of methods - procedures. Clin Exp Rheumatol 1989;7:213-18.

3 Vitali C, Moutsopoulos HM, Bombardieri S, et al. The European Study Group on diagnostic criteria in Sjögrens syndrome: sensitivity and specificity of tests for ocular and oral involvement in Sjögrens syndrome. Ann Rheum Dis 1994;53:637-47.

4 Cho P, Yap M. Schirmer Test - 11: A clinical study of its repeatability. Optom Vis Sci 1993;70:157-9.

5 Pastiides CA, Kitsios G, Karakostas KX, et al. Evaluation of tear break up time, Schirmer's-1 test and rose bengal staining as confirmatory tests for keratoconjunctivitis sicce. Clin ing as confirmatory tests for ker Exp Rheumatol 1989;7:155-7.

6 Heintze V, Birked D, Bjorn H. Secretion role and buffer effect of resting and stimulated whole saliva as a function of age and sex. Swed Dent J 1983;7:237-8

7 Vitali C, Bombardieri S, Moutsopoulos HM, et al. Preliminary criteria for the classification of Sjögrens syndrome: results of a prospective concerted action supported by the European Community. Arthritis Rheum 1993;36:340-7.

8 Vitali C, Bombardieri S, Moutsopoulos HM, et al. Assessment of the European Classification Criteria for Sjögrens syndrome in a series of clinically defined cases: results from a prospective multicentre study. Ann Rheum Dis 1996;55:116-21.

9 Drosos AA, Andropoulos AP, Costopoulos JS, Papadimitriou CS, Moutsopoulos HM. Prevalence of primary Sjögrens syndrome in an elderly population. Br J Rheumatol 1988;27:123-7.

10 Montecucco C, Bestagno M, Cerino A, Caporali R, Carnevale R, Longni M, et al. Anti SS-B/La antibodies in Sjögrens Syndrome and related autoimmune diseases: results of a quantitative immunoassay using a highly results of a quantitative immunoassay using a

11 Harley JB, Alexander E, Blas WB, et al. Anti Ro (SS-A) and Anti La (SS-B) in patients with Sjögrens syndrome. ArthriAnti La (SS-B) in patients with

12 Venables PJW, Shatties W, Peuse CT, et al. Anti La (SS-B): a diagnostic criterion for Sjögrens syndrome? Clin Exp Rheumatol 1989;7:181-4.

13 Jacobsson L, Hansen BU, Manthorpe R, et al. Association of dry eyes and dry mouth with anti Ro/SS-A and anti $\mathrm{La} / \mathrm{SS}-\mathrm{B}$ autoantibodies in normal adults. Arthritis Rheum 1992;35:1492-501. 